\title{
Characterization of Three Types of Quorum-Sensing Mutants in Burkholderia glumae Strains Isolated in Japan
}

\author{
Taro Kato ${ }^{1}$, Tomohiro Morohoshi ${ }^{1}$, Seiya Tsushima $^{2} \&$ Tsukasa Ikeda $^{1}$ \\ ${ }^{1}$ Department of Material and Environmental Chemistry, Graduate school of Engineering, Utsunomiya University, \\ Utsunomiya, Japan \\ ${ }^{2}$ National Institute of Agro-Environment Sciences, Natural Resource Inventory Center 3-1-3 Kannondai, Ibaraki, \\ Japan \\ Correspondence: Tsukasa Ikeda, Department of Material and Environmental Chemistry, Graduate School of \\ Engineering, Utsunomiya University, 7-1-2 Yoto, Utsunomiya, Japan. Tel: 81-28-689-6157. E-mail: \\ tikeda@cc.utsunomiya-u.ac.jp
}

Received: March 7, 2014 Accepted: April 23, 2014 Online Published: June 15, 2014

doi:10.5539/jas.v6n7p16 URL: http://dx.doi.org/10.5539/jas.v6n7p16

\begin{abstract}
Quorum sensing (QS) has been believed to be essential for the production of virulence factor such as toxoflavin, oxalate and the motility in Burkholderia glumae. Two types of QS mutant (tofI mutant) were recently reported in geographically different isolates of B. glumae. The tofI mutant derived from B. glumae BGR1 isolated in Korea had lost toxoflavin production but not the one derived from B. glumae 336gr-1 isolated in the United States. In the present study, we generated tofI mutants from 14 different B. glumae strains isolated in Japan and investigated the QS-regulated phenotypes. All tofI mutants failed to produce $N$-acyl- ${ }_{\mathrm{L}}$-homoserine lactones. However, tofI mutants from 11 out of 14 strains (Group I) retained toxoflavin productivity both on a solid and in a liquid medium like B. glumae 336gr-1 tofI mutant, whereas those from 2 strains (Group II) lost toxoflavin productivity both on a solid and in a liquid medium similar to B. glumae BGR1 tofI mutant. The other strain (Group III) showed novel phenotype because toxoflavin production was observed only on a solid medium. Furthermore, Group II tofI mutants lost oxalate productivity and exhibited a severe reduction in motility while Group I tofI mutants slightly produced oxalate and showed higher motility than Group II tofI mutants. These data suggest that the difference between Group I and II is not limited to toxoflavin production but also observed other QS-regulated functions. On the other hand, R1-types of morphological mutants frequently emerge from S-type colonies during the subculture for B. glumae (Tsushima et al., 1991). This study revealed that Group I wild-type strains displayed the S-type morphology and those of Group II showed the R1-type morphology. The results suggest that colony type is involved in the QS-regulated functions of Group I and II tofI mutants.
\end{abstract}

Keywords: Burkholderia glumae, quorum-sensing mutant, colony morphological mutant

\section{Introduction}

Burkholderia glumae is responsible for bacterial seedling rot and grain rot disease of rice and this pathogen causes serious damage to rice production in several countries (Goto \& Ohata., 1956; Cottyn et al., 1996a; Cottyn et al., 1996b; Jeong et al., 2003; Zeigler \& Alvares., 1989). The pathogenicity of B. glumae is associated with its multiplication on the grain after translocation from the leaf sheath (Tsushima., 1996; Tsushima et al., 1987). The tofI/tofR quorum-sensing (QS) system of B. glumae plays a central role in its pathogenicity (Kim et al., 2004). QS is a gene regulation system depending on cell density in bacteria (Fuqua et al., 1996). In several gram-negative bacteria including $B$. glumae, $N$-acyl- ${ }_{-}-$homoserine lactone (AHL) is used as a $Q S$ signal molecule in this system. AHL is constitutively synthesized by a member of the LuxI protein family and the concentration increases with local cell-density. When AHL concentration reaches a threshold, they form complex with the AHL receptor protein belonging to the LuxR protein family and bind certain sequence called lux-box like sequence, resulting in coordinated various gene expressions. In B. glumae, tofI encodes a AHL synthase responsible for the production of $N$-octanoyl-L-homoserine lactone (C8-HSL) and

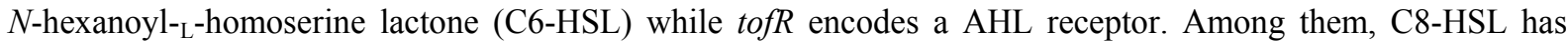
been identified as the main QS signal molecule in B. glumae. B. glumae produces phytotoxins including fervenulin, reumycin and toxoflavin (Kim et al., 2004). In the B. glumae BGR1 strain isolated in Korea, 
disruption of tofI resulted in the loss of toxoflavin production because the C8-HSL-TofR complex activates toxJ, which is responsible for the expression of the tox operon required for toxoflavin biosynthesis and secretion (Kim et al., 2004). Furthermore, QS regulates the expression of the gene encoding an IclR-type transcriptional regulator, qsmR (Kim et al., 2007), which regulates oxalate production (Goo et al., 2012), catalase activity (Chun et al., 2009), motility (Kim et al., 2007) and the type II secretion system (Goo et al., 2011). Because QS is essential for rice spikelet pathogenicity in B. glumae BGR1 (Kim et al., 2007), it is expected to become one of the main targets for antivirulence therapy and to replace the current antimicrobials for B. glumae. However, the tofI mutant of B. glumae 336gr-1, isolated in the United States, retains a high level of toxoflavin productivity and pathogenicity on spikelets, despite the loss of AHL productivity (Chen et al., 2012). Although it is unknown what cause the discrepant results between BGR1 and 336gr-1, it will become an important research for $B$. glumae to understand phenotypic and genetic diversity among strains. Since BGR1 and 336gr-1 are geographically distinct isolates, we focussed on the role of QS in B. glumae strains isolated in Japan because bacterial seedling rot and grain rot disease of rice by B. glumae was first reported in Japan (Uematsu et al., 1976; Goto \& Ohata., 1956). In this study, we constructed and characterized the QS mutants of the B. glumae strains isolated in Japan.

\section{Materials and Methods}

\subsection{Bacterial Strains, Plasmids and Chemicals}

We used 14 B. glumae strains isolated from various sites on rice or bean sprouts at several locations in Japan from 1979 to 2005 (Table 1). The other bacterial strains and plasmids used in this study are listed in Table 2. Luria-Bertani (LB) medium was used as the routine bacterial growth medium (Sambrook et al., 1989) and potato semisynthetic agar (PSA) medium (Yoshida et al., 2001) was used to study colony morphology Both C6-HSL and C8-HSL were synthesized as previously described (Ikeda et al., 2001). Antibiotics were added with final concentrations of $100 \mu \mathrm{g} / \mathrm{ml}$ ampicillin, $50 \mu \mathrm{g} / \mathrm{ml}$ kanamycin and $10 \mu \mathrm{g} / \mathrm{ml}$ collistin.

\subsection{Construction of the tofI Mutants}

We constructed a marker-less tofI mutant by site-directed mutagenesis using inverse PCR (Dorrell et al., 1996). An approximately 2300-bp sequence containing the tofI region was amplified by PCR using KOD FX polymerase (Toyobo Life Science, Japan). The forward and reverse primers tofI-1000-F (5'-TGACGTCGCGATCCTCTGTCTGC-3') and tofI-1000-R (5'-GAGCTGCTGCTCGTTTTCCGCC-3'), respectively, were designed based on the B. glumae BGR1 genome (Lim et al., 2009). Chromosomal DNA extracted from MAFF 302748 served as a template under the following reaction conditions: $94{ }^{\circ} \mathrm{C}$ for 2 min, followed by 27 cycles at $98^{\circ} \mathrm{C}$ for $10 \mathrm{~s}$ and $68^{\circ} \mathrm{C}$ for $2.5 \mathrm{~min}$. The PCR fragment was inserted into the pGEM-T Easy Vector using TArget Clone -Plus- (Toyobo Life Science, Japan) and Ligation high Ver2 (Toyobo Life Science, Japan) to generate pMtofI1000. Furthermore, we designed two primers in opposite orientations at the mutation site: tofI-N, 5'-CATTCGCATCGCGTCCCAATACGT-3' and tofI-C, 5'-TCACGTTCTGCAGCATCGAGCG-3'. Inverse PCR was performed with the primer set, KOD FX polymerase and pMtofl1000 as template under the following reaction conditions: $94{ }^{\circ} \mathrm{C}$ for $2 \mathrm{~min}$, followed by 10 cycles at $98{ }^{\circ} \mathrm{C}$ for $10 \mathrm{~s}$ and $68^{\circ} \mathrm{C}$ for $5 \mathrm{~min}$. After amplification, the remaining plasmid DNA template was removed by digestion with DpnI and self-ligated to generate the pMtofI 800 vector. The kanamycin resistance cassette was cut out of the pKPR11 vector using PstI and introduced into the PstI site of the pGP704Sac38 suicide vector, which contains sucrose-sensitive gene $s a c B$, to generate pGPSacBKm. The pMtofI800 vector was digested with EcoRI and the fragment containing the disrupted tofI was inserted into the MfeI site of pGPSacBKm to generate pGPSacBtofI800. The disruption of tofI was performed by bacterial conjugation and homologous recombination. Conjugation was conducted between each strain of B. glumae and Escherichia coli S17-1 $\lambda$ pir carrying pGPSacBtofi800. After incubation in LB agar containing $10 \mathrm{mM} \mathrm{MgSO}{ }_{4}$ at $30{ }^{\circ} \mathrm{C}$ for $4 \mathrm{~h}$, the cells were streaked on LB agar containing $50 \mu \mathrm{g} / \mathrm{ml}$ kanamycin and $10 \mu \mathrm{g} / \mathrm{ml}$ collistin (all B. glumae strain tolerant to collistin) and incubated at $37^{\circ} \mathrm{C}$ for $48 \mathrm{~h}$. This medium is sensitive for $E$. coli S17-1 $\lambda$ pir but resistant for B. glumae. Single colonies were picked up with a toothpick, transferred to $4 \mathrm{ml}$ of LB broth and incubated at $37^{\circ} \mathrm{C}$ for $16 \mathrm{~h}$ with shaking. After incubation, the culture was streaked on LB agar plates containing $10 \%$ sucrose and incubated at $37{ }^{\circ} \mathrm{C}$ for $24 \mathrm{~h}$. The disruption of tofI was confirmed by AHL productivity and by the band size of the PCR product amplified with the tofI (H)-F primer 5'-GTTCGTCAACGACGACTACG-3' and tofI (H)-R primer 5'-CGGAATTACCACGAGGACAC-3' (Chen et al., 2012).

\subsection{Detection of AHL Productivity}

We investigated the different types of AHL produced by the wild-type strains using thin-layer chromatography (Shaw et al., 1997) using TLC plate (RP-18 F254S, Merck) and C. violaceum CV026 as a biosensor, which 
clearly distinguishes C6-HSL and C8-HSL (McClean et al., 1997). The AHL production by tofI mutants was investigated by cross-streak using C. violaceum CV026 and VIR07 (Morohoshi et al., 2008). C. violaceum VIR07 shows stronger responses to C8-HSL but not to C6-HSL than CV026. These biosensors response to exogenous AHLs by producing the purple pigment violacein.

\subsection{Characterization of the QS-Regulated Functions}

We investigated toxoflavin and oxalate productivity as well as the motility of the 14 wild-type strains and mutants. Toxoflavin appears as a yellow pigment in LB medium (Chen et al., 2012; Kim et al., 2004). Therefore, each strain was streaked on LB agar plates with or without $100 \mathrm{nM} \mathrm{C} 8$-HSL and incubated at $37{ }^{\circ} \mathrm{C}$ for $24 \mathrm{~h}$. Toxoflavin productivity was assessed by the intensity of the yellow pigment in solid culture. Furthermore, we investigated toxoflavin productivity in liquid culture. Fourty microlitter of each cell suspension $\left(\mathrm{OD}_{610}=0.2\right)$ was inoculated to $4 \mathrm{ml}$ of LB broth and incubated at $37{ }^{\circ} \mathrm{C}$ for $24 \mathrm{~h}$ with shaking. After incubation, toxoflavin was extracted from culture supernatant with equal volume of chloroform and dried in draft chamber for a night. The extraction was resuspended in $100 \mu \mathrm{l}$ of methanol and toxoflavin productivity was assessed by the intensity of the yellow pigment. For the oxalate assay, $5 \mu$ l of each cell suspension was added to LB agar containing $0.1 \%$ $\mathrm{CaCl}_{2}$ (Li et al., 1999) with or without $100 \mathrm{nM} \mathrm{C} 8$-HSL, and incubated at $37{ }^{\circ} \mathrm{C}$ for $24 \mathrm{~h}$. Oxalate productivity was positive when calcium oxalate crystals were observed in the medium using light microscopy (Olympus SZ61, Olympus Corporation, Tokyo, Japan). Motility was investigated as previously described, with some modifications (Kato et al., 2013). Each cell suspension was deposited at the centre of a $90 \varphi \times 15 \mathrm{~mm}$ petri dish containing $0.3 \% \mathrm{LB}$ agar and incubated at $37{ }^{\circ} \mathrm{C}$ for $48 \mathrm{~h}$. Motility was investigated three times and assessed by measuring colony diameter.

\subsection{Colony Morphology}

We investigated colony morphology of the wild-type strains on PSA medium. The colony type was divided into S-type (smooth, white, shiny, dome) or R1-type (rough, translucent, flat), as previously described (Tsushima et al., 1991).

Table 1. Burkholderia glumae strains isolated in Japan

\begin{tabular}{ccccc}
\hline Burkholderia glumae & $\begin{array}{c}\text { Isolation } \\
\text { date }\end{array}$ & Source & $\begin{array}{c}\text { Site on the } \\
\text { source }\end{array}$ & Location \\
\hline MAFF 301441 & 1982 & Oryza sativa & Unknown & Hiroshima, Japan \\
MAFF 302417 & 1984 & Vigna radiate & Sprout & Tokyo, Japan \\
MAFF 302437 & 1984 & Oryza sativa & Grain & Ooita, Japan \\
MAFF 302552 & 1991 & Oryza sativa & Leaf sheath & Kumamoto, Japan \\
MAFF 302748 & 1982 & Oryza sativa & Seedling & Ibaraki, Japan \\
MAFF 302874 & 1979 & Oryza sativa & Grain & Ibaraki, Japan \\
MAFF 302930 & 1990 & Oryza sativa & Seedling & Iwate, Japan \\
MAFF 302934 & 1990 & Oryza sativa & Seedling & Miyagi, Japan \\
MAFF 311026 & 1991 & Oryza sativa & Seedling & Hokkaido, Japan \\
MAFF 311193 & 1997 & Oryza sativa & Seedling & Toyama, Japan \\
MAFF 311196 & 1996 & Oryza sativa & Seedling & Nagano, Japan \\
MAFF 311199 & 1996 & Oryza sativa & Seedling & Yamaguchi, Japan \\
MAFF 311266 & 1982 & Oryza sativa & Grain & Fukuoka, Japan \\
MAFF 311509 & 2005 & Oryza sativa & Grain & Okinawa, Japan \\
\hline
\end{tabular}


Table 2. Bacterial strains and plasmids

\begin{tabular}{|c|c|c|}
\hline Strains & Description & Source or reference \\
\hline \multicolumn{3}{|l|}{ Escherichia coli } \\
\hline S17-1 $\lambda /$ pir & $\begin{array}{c}\text { thi pro hsdR hsdM+ recA RP4 } \\
2 \mathrm{Rc}:: \mathrm{Mu}-\mathrm{Km}:: \operatorname{Tn} 7\end{array}$ & Simon et al., 1983 \\
\hline \multicolumn{3}{|c|}{ Burkholderia glumae } \\
\hline dI441 & A $\Delta$ tofI derivative of MAFF 301441 & This study \\
\hline dI417 & A $\Delta$ tofI derivative of MAFF 302417 & This study \\
\hline dI 437 & A $\Delta$ tofI derivative of MAFF 302437 & This study \\
\hline dI552 & A $\Delta$ tofI derivative of MAFF 302552 & This study \\
\hline dI748 & A $\Delta$ tofI derivative of MAFF 302748 & This study \\
\hline dI874 & A $\Delta$ tofI derivative of MAFF 302874 & This study \\
\hline dI930 & A $\Delta$ tofI derivative of MAFF 302930 & This study \\
\hline dI934 & A $\Delta$ tofI derivative of MAFF 302934 & This study \\
\hline dI026 & A $\Delta$ tofI derivative of MAFF 311026 & This study \\
\hline dI193 & A $\Delta$ tofI derivative of MAFF 311193 & This study \\
\hline dI196 & A $\Delta$ tofI derivative of MAFF 311196 & This study \\
\hline dI199 & A $\Delta$ tofI derivative of MAFF 311199 & This study \\
\hline dI266 & A $\Delta$ tofI derivative of MAFF 311266 & This study \\
\hline dI509 & A $\Delta$ tofI derivative of MAFF 311509 & This study \\
\hline \multicolumn{3}{|c|}{ Chromobacterium violaceum } \\
\hline CV026 & $\begin{array}{l}\text { ATCC } 31532 \text { derivative, cviI::Tn5sylE, } \\
\text { Kmr }^{\mathrm{r}}, \mathrm{Sm}^{\mathrm{r}}\end{array}$ & McClean et al., 1997 \\
\hline VIR07 & $\begin{array}{c}\text { ATCC } 12472 \text { derivative, cviI:: } \mathrm{Km}^{\mathrm{r}} \text {, } \\
\text { Amp }^{\mathrm{r}}\end{array}$ & Morohoshi et al., 2008 \\
\hline \multicolumn{3}{|l|}{ Plasmids } \\
\hline pGEM-T easy & Cloning vector, $\mathrm{Amp}^{\mathrm{r}}$ & Promega \\
\hline pMtofI1000 & pGEM-T containing tofI, Amp ${ }^{\mathrm{r}}$ & This study \\
\hline pMtofI 800 & pGEM-T containing $\Delta t o f I, \mathrm{Amp}^{\mathrm{r}}$ & This study \\
\hline pKRP11 & Cloning vector, $\mathrm{Km}^{\mathrm{r}}, \mathrm{Amp}^{\mathrm{r}}$ & Reece \& Phillips., 1995 \\
\hline pGP704Sac38 & $\begin{array}{l}\text { pBR322 derivative with R6K ori, mob } \\
\text { RP4, polylinker from M13 tg131 } \\
\text { containing } s a c B, \mathrm{Amp}^{\mathrm{r}}\end{array}$ & Morohoshi et al., 2004 \\
\hline pGPSacBKm & pGP704Sac38 inserted $\mathrm{Km}^{\mathrm{r}}$ gene & This study \\
\hline pGPSacBtofI 800 & pGPSacBKm containing $\Delta$ tofI, $\mathrm{Km}^{\mathrm{r}}$ & This study \\
\hline
\end{tabular}

\section{Results}

\section{1 tofl Disruption Prevents AHL Production}

We characterized the different types of AHLs produced by each B. glumae strain and confirmed that all the wild-type strains produced C8-HSL and C6-HSL (Table 3). Furthermore, we constructed a marker-less tofI mutant for each strain and confirmed that all tofI mutants failed to produce AHLs (Table 3). The size of the PCR fragment amplified with tofI $(\mathrm{H})$ primer sets was as expected (data not shown). 
Table 3. Productivity of AHL, toxoflavin, oxalate and colony morphology for Burkholderia glumae wild-type strains isolated in Japan and their tofI mutants

\begin{tabular}{|c|c|c|c|c|c|c|}
\hline \multirow{4}{*}{$\begin{array}{c} \\
\text { MAFF } 301441 \\
\text { dI4441 }\end{array}$} & \multirow{2}{*}{$\begin{array}{c}\text { AHL } \\
\text { productivity }\end{array}$} & \multicolumn{2}{|c|}{ Toxoflavin productivity } & \multirow{2}{*}{$\begin{array}{c}\text { Oxalate } \\
\text { productivity }\end{array}$} & \multirow{2}{*}{$\begin{array}{c}\text { Colony } \\
\text { morphology }\end{array}$} & \multirow{2}{*}{ Group } \\
\hline & & Solid culture & Liquid culture & & & \\
\hline & C6-HSL, C8-HSL & + & + & ++ & $\mathrm{S}^{\mathrm{a}}$ & \multirow{2}{*}{ I } \\
\hline & - & + & + & + & $\mathrm{ND}^{\mathrm{b}}$ & \\
\hline MAFF 302417 & C6-HSL, C8-HSL & + & + & ++ & $\mathrm{R} 1^{\mathrm{c}}$ & \multirow{2}{*}{ II } \\
\hline dI417 & - & - & - & - & ND & \\
\hline MAFF 302437 & C6-HSL, C8-HSL & + & + & ++ & S & \multirow{2}{*}{ I } \\
\hline dI437 & - & + & + & + & ND & \\
\hline MAFF 302552 & C6-HSL, C8-HSL & + & + & ++ & $\mathrm{S}$ & \multirow{2}{*}{ I } \\
\hline dI552 & - & + & + & + & ND & \\
\hline MAFF 302748 & C6-HSL, C8-HSL & + & + & ++ & $\mathrm{S}$ & \multirow{2}{*}{ I } \\
\hline dI 748 & - & + & + & + & ND & \\
\hline MAFF 302874 & C6-HSL, C8-HSL & + & + & ++ & $\mathrm{R} 1$ & \multirow{2}{*}{ II } \\
\hline dI874 & - & - & - & - & ND & \\
\hline MAFF 302930 & C6-HSL, C8-HSL & + & + & ++ & S & \multirow{2}{*}{ I } \\
\hline dI930 & - & + & + & + & ND & \\
\hline MAFF 302934 & C6-HSL, C8-HSL & + & + & ++ & $\mathrm{S}$ & \multirow{2}{*}{ III } \\
\hline dI934 & - & + & - & + & ND & \\
\hline MAFF 311026 & C6-HSL, C8-HSL & + & + & ++ & $\mathrm{S}$ & \multirow{2}{*}{ I } \\
\hline dI026 & - & + & + & + & ND & \\
\hline MAFF 311193 & C6-HSL, C8-HSL & + & + & ++ & S & \multirow{2}{*}{ I } \\
\hline dI193 & - & + & + & + & ND & \\
\hline MAFF 311196 & C6-HSL, C8-HSL & + & + & ++ & S & \multirow{2}{*}{ I } \\
\hline dI196 & - & + & + & + & ND & \\
\hline MAFF 311199 & C6-HSL, C8-HSL & + & + & ++ & S & \multirow{2}{*}{ I } \\
\hline dI199 & - & + & + & + & ND & \\
\hline MAFF 311266 & C6-HSL, C8-HSL & + & + & ++ & $\mathrm{S}$ & \multirow{2}{*}{ I } \\
\hline $\mathrm{d} / 266$ & - & + & + & + & ND & \\
\hline MAFF 311509 & C6-HSL, C8-HSL & + & + & ++ & S & \multirow{2}{*}{ I } \\
\hline dI509 & - & + & + & + & ND & \\
\hline
\end{tabular}

${ }^{a}$ S-type colony morphology ${ }^{b}$ Not determined ${ }^{c}$ R1-type colony morphology.

\section{2 Strain-Specific QS Dependency for Toxoflavin and Oxalate Production}

All the wild-type strains possessed toxoflavin production both on a solid and in a liquid medium (Table 3). As the result of tofI disruption, among the marker-less tofI mutants, 11 out of 14 strains (Group I) retained toxoflavin productivity both on a solid medium and in a liquid medium similar to B. glumae 336gr-1 (Figure 1A, B). Two strains, dI417 and dI874 (Group II) lost the ability to produce toxoflavin both on a solid medium and in a liquid medium, like B. glumae BGR1 (Figure 1A, B). Furthermore, dI934 (Group III) was able to produce toxoflavin on solid medium but not in liquid medium (Figure 1A, B).

\section{3 Evidence for QS Dependent and Independent Motility}

The Group I and III tofI mutants showed equal level of or slightly lower motility with that of their wild-type strains (Figure 3A). The loss of toxoflavin and oxalate productivity in the Group II tofI mutants was associated with a severe reduction in motility compared with their wild-type strains and Group I and III tofI mutants (Figure 3A, B). The Group II wild-type strains showed greater motility than that of the Group I and III wild-type strains (Figure 3B), suggesting that other factors affect motility. Furthermore, the addition of C8-HSL dramatically restored the motility in the dI874 mutant of Group II (Figure 3C). 


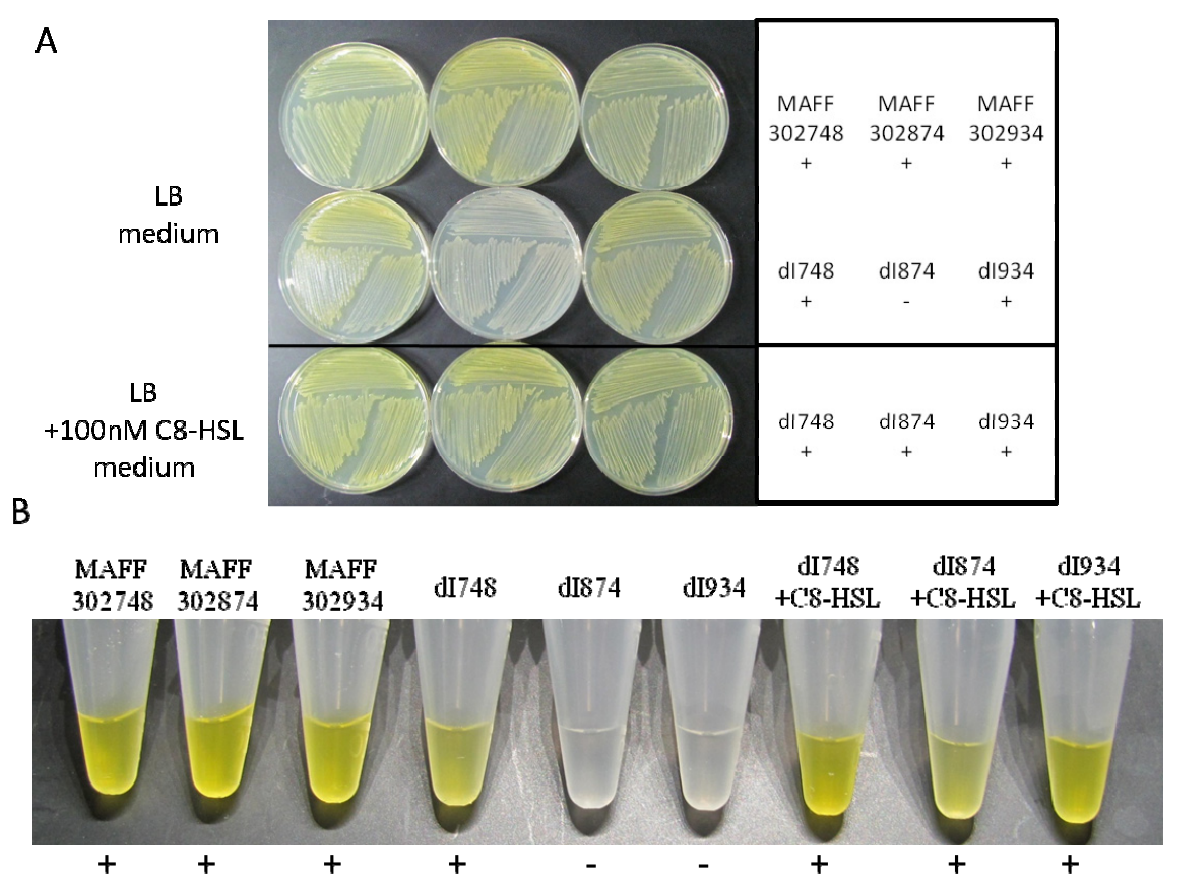

Figure 1. Toxoflavin productivity of the wild-type strains MAFF 302748,MAFF 302874, MAFF 302934 and their respective tofI mutants dI748, dI874 and dI934 on LB agar (A) or in LB broth (B). Each strain was streaked on LB agar or cell suspension $\left(\mathrm{OD}_{610}=0.2\right)$ was inoculated to $\mathrm{LB}$ broth and incubated at $37^{\circ} \mathrm{C}$ for $24 \mathrm{~h}$ with or without $100 \mathrm{nM}$ C8-HSL. After incubation, toxoflavin productivity was assessed by the productivity of yellow pigment in agar or concentrated chloroform extraction from liquid culture

All the wild-type strains exhibited oxalate productivity (Table 3). As the result of tofI disruption, oxalate calcium crystals were observed in Group I and III but not detected in Group II (Table 3, Figure 2). The amount of oxalate for Group I and III tofI mutants appeared to be lower than that for the wild-type strain. This is because oxalate calcium crystals were observed inside and outside the wild-type colonies but only inside the tofI mutant colonies (Figure 2, Table 3). The addition of C8-HSL restored toxoflavin and oxalate productivity in the dI874 mutant of Group II (Figures 1A, B and 2), essentially bypassing the loss of tofI-dependent AHL synthase expression.

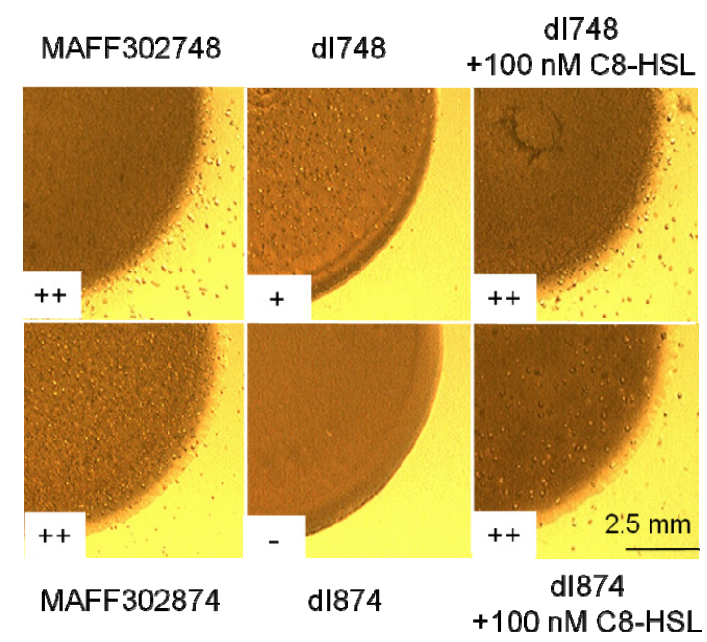

Figure 2. Oxalate productivity of the wild-type strains MAFF 302748 and MAFF 302874 and their respective tofI mutant dI 748 and dI874. Each strain was grown on LB agar containing $0.1 \% \mathrm{CaCl}_{2}$ with or without $100 \mathrm{nM}$ C8-HSL at $37{ }^{\circ} \mathrm{C}$ for $24 \mathrm{~h}$. Oxalate productivity was detected by the presence of calcium oxalate crystals in inside and/or outside of the colony using light microscopy 


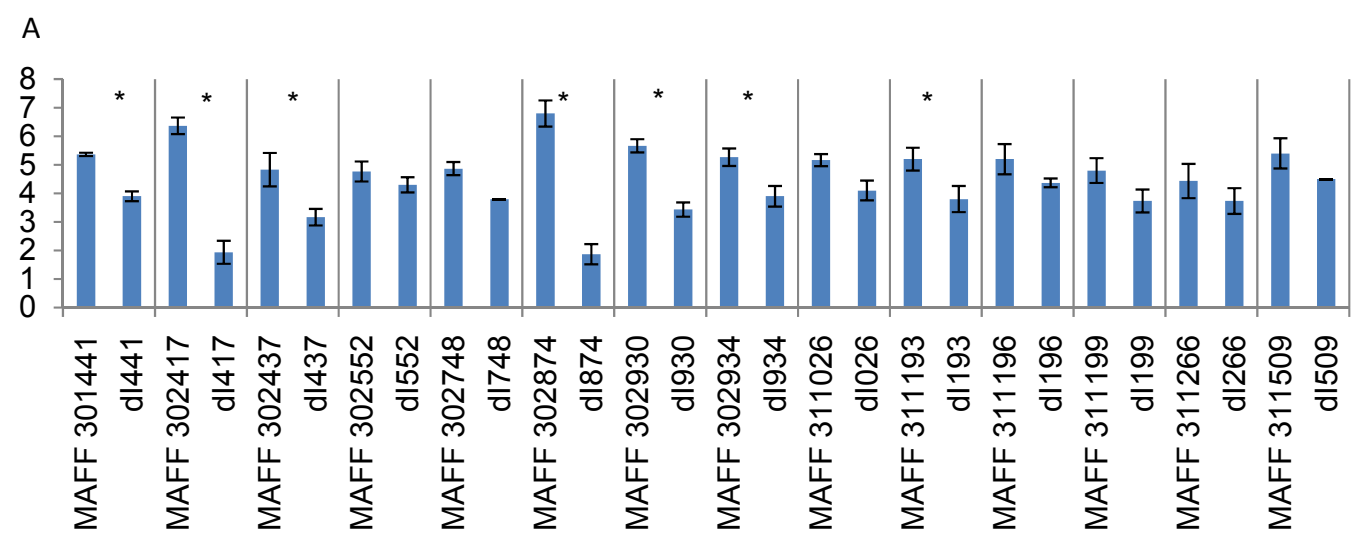

B
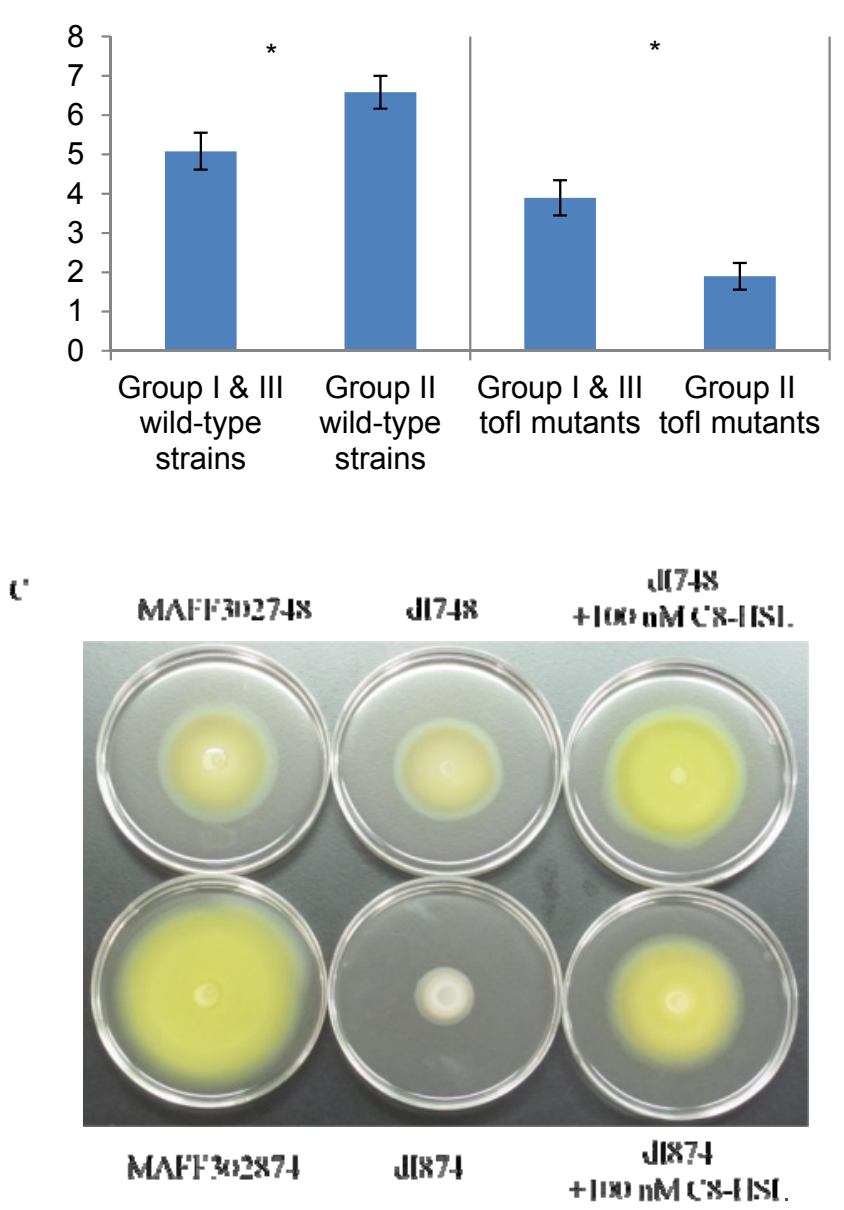

Figure 3. Motility of B. glumae wild-type strains isolated in Japan and respective tofI mutants on $0.3 \%$ LB agar medium with or without $100 \mathrm{nM} \mathrm{C} 8$-HSL at $37{ }^{\circ} \mathrm{C}$ for $48 \mathrm{~h}$. (A) Comparison of motility between the wild-type strain and tofI mutant. (B) Comparison of average of colony diameter between Group I, III and II wild-type strains or tofI mutants. Bars on columns indicate that standard deviations. Differences in the motility were determined by Tukey-Kramer multiple comparisons method. * indicates significant differences between each strain or groups (P, 0.01). (C) Motility of the wild-type strains MAFF 302748 and MAFF 302874 and respective tofI mutant dI748 and dI874 with or without $100 \mathrm{nM} \mathrm{C8-HSL}$

\subsection{Different Colony Morphology Among Group I and II Wild-Type Strains}

All Group I and III wild-type strains showed smooth, white, shiny and dome-type colony morphology (S-type colony) (Figure 4). On the other hand, we could not detect the S-type colony in Group II wild-type strains. 
Instead, they presented rough, translucent and flat colony morphology (Figure 4), which is typical of the R1-type colony that emerged during subculture of the S-type strain of B. glumae (Tsusima et al., 1991).

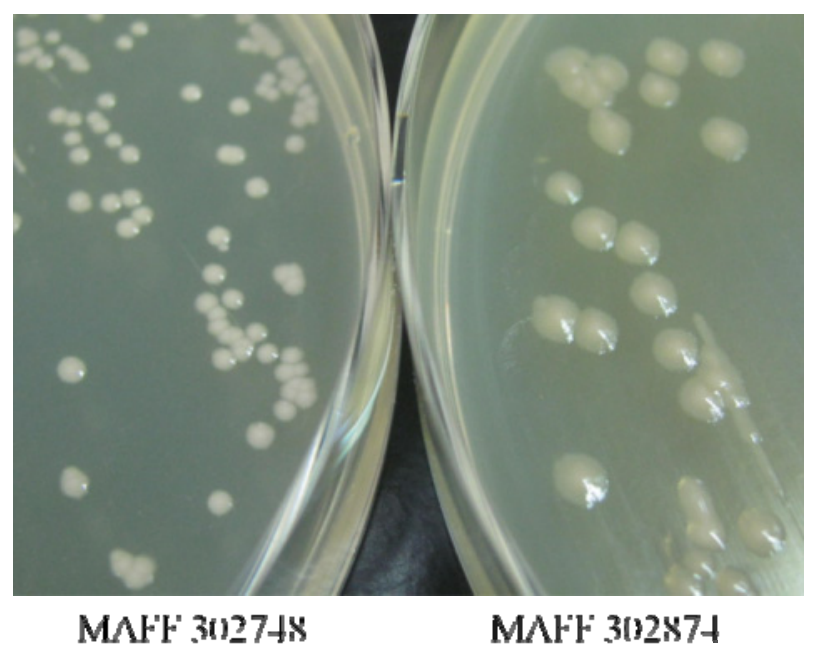

Figure 4. Colony morphology of the wild-type strains MAFF 302748 and MAFF 302874. Each strain was streaked on potato semisynthetic agar (PSA) medium and incubated at $30^{\circ} \mathrm{C}$ for four days

\section{Discussion}

Two B. glumae QS mutants with distinct pathogenicity were reported in Korea (B. glumae BGR1; Kim et al., 2004) and the United States (B. glumae 336gr-1; Chen et al., 2012). Although both presented mutations in the tofI gene, which is responsible for toxoflavin production, B. glumae 336gr-1 retained toxoflavin-related pathogenicity. The present study was designed to investigate the causes of this discrepancy to improve our understanding of the regulation mechanisms of virulence-related functions and strain diversity for B. glumae.

We constructed and characterized 14 QS tofI mutant B. glumae strains isolated from various regions in Japan. As expected, all QS mutants failed to produce AHLs (Table 3). However, 12 mutant strains (Group I and III) maintained toxoflavin productivity on a solid medium like the American B. glumae 336gr-1 strain, whereas two strains (Group II) lost the productivity similar to Korean B. glumae BGR1 (Table 3). These data suggested the existence of tofI-independent mechanisms for the regulation of toxoflavin, as previously suggested for $336 \mathrm{gr}-1$ (Chen et al., 2012). Furthermore, not only Group II tofI mutant but also Group III dI934 did not produce toxoflavin in liquid culture (Table 3, Figure 1B). Although we reisolated dI934 from liquid culture and inoculated them to solid medium, they retained toxoflavin productivity on solid medium (data not shown), suggests that the loss of toxoflavin production by dI934 is specific to liquid culture. The amount of toxoflavin produced by $q s m R$ mutant increases until $12 \mathrm{~h}$ after incubation but then decrease after $16 \mathrm{~h}$ in liquid medium but not on solid medium (Kim et al., 2007). Thus, it will be necessary to investigate the relationship between incubation times and toxoflavin productivity. In addition, we demonstrated that other QS-regulated functions, such as oxalate production and motility, of the tofI mutants were still preserved on the equal or slightly lower level with the wild-type strains in Group I and III but severe reduction of these functions was observed in Group II (Table 3, Figures 3A, B). Toxoflavin production is directly regulated by QS (Kim et al., 2004), whereas oxalate production and motility are indirectly regulated by QS via QsmR (Goo et al., 2012; Kim et al., 2007). Therefore, unknown additional regulators may be involved in not only toxoflavin production but also other QS-regulated functions in Group I and III.

The addition of C8-HSL was able to recover the functions of Group II and III tofI mutant to almost equal level with the wild-type strain (Figures 1, 2, 3C), suggesting that secondary mutations in other important sites for these functions during allelic-exchange procedure as previously observed in Pseudomonas aeruginosa QS mutants (Beaston et al., 2002) is not occurred.

Phylogenetic analysis based on $g y r B$ and $r p o D$ sequences revealed a limited diversity of the Japanese strains of $B$. glumae compared with other Burkholderia plant pathogens and that the two members of Group II (MAFF 302417 and MAFF 302874) were in the same cluster as Group I strains, MAFF 302552 (Maeda et al., 2006). 
This indicates that the differences between Group I and II are not associated with phylogenetic associations based on housekeeping genes.

For several years, QS has been considered to be essential for the expression of virulence-related functions and for the plant and human pathogenicity of Burkholderia species (Kim et al., 2004; Ulrich et al., 2004). A recent study showed that the B. mallei GB8 and ATCC 23344 QS mutant exhibited a pathogenicity comparable with that the parent strain in an aerosolized mouse infection model (Majerczyk et al., 2013) contrary to the previous report in other B. mallei QS mutant (Ulrich et al., 2004). As one possibility, the authors suggested that laboratory passages and the accumulation of spontaneous mutations select these different types of QS mutants because the genome of $B$. mallei is plastic (Nierman et al., 2004) and genome variability between passages is considered to be a feature of $B$. mallei (Romero et al., 2006). Similarly, natural mutants of $B$. glumae frequently emerge during subculture and rice infection (Tsushima et al., 1991; Kato et al., 2013; Nandakumar et al., 2009). It is reported that there are three types of colony morphological mutants (CMMs) from S-type colony strains of MAFF 302748 during subculture: R1-1, R1-2 and R2 (Kato et al., 2013). In this study, all members of Group I and III wild-type strains showed an S-type colony morphology, whereas both Group II wild-type strains showed an R1-type colony morphology (Figure 4). There are possibilities that the Group II strains experienced genomic mutations during laboratory passage. However, the previous isolates of CMM showed lower motility than the wild-type strain (Kato et al., 2013), whereas the Group II strains were extremely motile (Figure 3C). Thus, the previous CMM isolates and the Group II wild-type strains may represent different types although they share the R1-type colony morphology.

The commercial type strain ATCC 33617 of B. glumae lost its toxoflavin productivity and pathogenicity on rice spikelets because of frameshift mutations in tofR (Devescovi et al., 2007). tofR complementation restored toxoflavin production and pathogenicity, which suggests that type strain ATCC 33617 may belong in Group II. However, additional mutations may exist because mutation sites are not limited to one place in laboratory strains (Beaumont et al., 2009).

Mutable functions not associated with the phylogenetic relationship are common features of B. glumae, which makes it difficult to elucidate the mechanisms supporting virulence. It will be important to understand phenotypic and genetic diversity among inter-strains in future studies for B. glumae. Although the relationship between colony morphological change and the generation of Group I and II remains poorly understood, it may be necessary to consider the generation of intra-strain variation to shape strain diversity for B. glumae.

\section{References}

Beatson, S. A., Whitchurch, C. B., Semmler, A. B. T., \& Mattick, J. S. (2002). Quorum sensing is not required for twitching motility in Pseudomonas aeruginosa. Journal of Bacteriology, 184, 3598-3604. http://dx.doi.org/10.1128/JB.184.13.3598-3604.2002

Beaumont, H. J. E., Gallie, J., Kost, C., Gerguson, G. C., \& Rainey, P. B. (2009). Experimental evolution of bet hedging. Nature, 462, 90-94. http://dx.doi.org/10.1038/nature08504

Chen, R., Barphagha, I. K., Karki, H. S., \& Ham, J. H. (2012). Dissection of quorum-sensing genes in Burkholderia glumae reveals non-canonical regulation and the new regulatory gene tof $M$ for toxoflavin production. Plos One, e52150. http://dx.doi.org/10.1371/journal.pone.0052150

Chun, H., Choi, O., Goo, E., Kim, N., Kim, H., Kang, Y., ... Hwang, I. (2009). The quorum sensing-dependent gene kat $G$ of Burkholderia glumae is important for protection from visible light. Journal of Bacteriology, 13, 4152-4157. http://dx.doi.org/10.1128/JB.00227-09

Cottyn, B., Cerez, M. T., Outryve, M. F., \& Barroga, J. (1996a). Bacterial diseases of rice 1. Pathogenic bacteria associated with sheath rot complex and grain discoloration of rice in the Philippines. Plant Disease, 80, 429-437. http://dx.doi.org/10.1094/PD-80-0429

Cottyn, B., Outryve, M. F., Cerez, M. T., Cleene, M. D., \& Swings, J. (1996b). Bacterial diseases of rice 2. Characterization of pathogenic bacteria associated with sheath rot complex and grain discoloration of rice in the Philippines. Plant Disease, 80, 438-445. http://dx.doi.org/10.1094/PD-80-0438

Devescovi, G., Bigirimana, J., Degrassi, G., Cabrio, L., LiPuma, J. J., Kim, J., ... Venturi, V. (2007). Involvement of a quorum-sensing-regulated lipase secreted by a clinical isolate of Burkholderia glumae in severe disease symptoms in rice. Applied and Environmental Microbiology, 73, 4950-4958. http://dx.doi.org/10.1128/AEM.00105-07

Dorrell, N., Gyselman, V. G., Foynes, S., Li, S. R., \& Wren, B. W. (1996). Improved efficiency of inverse PCR mutagenesis. Biotechniques, 21, 604-608. 
Fuqua, C., Winans, S. C., \& Greenberg, E. P. (1996). Census and consensus in bacterial ecosystems: The LuxR-LuxI family of quorum-sensing transcriptional regulators. Annual Review of Microbiology, 50, 727-751. http://dx.doi.org/10.1146/annurev.micro.50.1.727

Goo, E, Kang, Y., Kim, H., \& Hwang, I. (2010). Proteomic analysis of quorum sensing-dependent proteins in Burkholderia glumae. Journal of Proteome Research, 9, 3184-3199. http://dx.doi.org/10.1021/pr100045n

Goo, E., Majerczyk, C. D., An, J. H., Chandler, J. R., Seo, Y. S., Ham, H., ... Hwang, I. (2012). Bacterial quorum sensing, cooperativity, and anticipation of stationary-phase stress. Proceedings of the National Academy of Sciences of the United States of America, 48, 19775-19780. http://dx.doi.org/10.1073/pnas.1218092109

Goto, K., \& Ohata, K. (1956). New bacterial diseases of rice (brown stripe and grain rot). Annals of the Phytopathological Society of Japan, 21, 46-47.

Ikeda, T., Kajiyama, K., Kita, T., Takiguchi, N., Kuroda, A., Kato, J., \& Ohtake, H. (2001). The synthesis of optically pure enantiomers of $\mathrm{N}$-acyl-homoserine lactone autoinducers and their analogues. Chemistry Letters, 30, 314-315. http://dx.doi.org/10.1246/cl.2001.314

Jeong, Y., Kim, J., Kim, S., Kang, Y., Nagamatsu, T., \& Hwang, I. (2003). Toxoflavin produced by Burkholderia glumae causing rice grain rot is responsible for inducing bacterial wilt in many field crops. Plant Disease, 87, 890-895. http://dx.doi.org/10.1094/PDIS.2003.87.8.890

Kato, T., Morohoshi, T., Tsushima, S., \& Ikeda, T. (2013). Phenotypic characterization of colony morphological mutants of Burkholderia glumae that emerged during subculture. Journal of General Plant Pathology, 79, 249-259. http://dx.doi.org/10.1007/s10327-013-0454-6

Kim, J., Kang, Y., Choi, O., Jeong, Y., Jeong, J. Y., Lim, J. Y., ... Hwang, I. (2007). Regulation of polar flagellum genes is mediated by quorum sensing and FlhDC in Burkholderia glumae. Molecular Microbiology, 64, 165-179. http://dx.doi.org/10.1111/j.1365-2958.2007.05646.x

Kim, J., Kim, J. G., Kang, Y., Jang, J. Y., Jog, J. G., Lim, J. Y., ... Hwang I. (2004). Quorum sensing and the LysR-type transcriptional activator ToxR regulate toxoflavin biosynthesis and transport in Burkholderia glumae. Molecular Microbiology, 54, 921-934. http://dx.doi.org/10.1111/j.1365-2958.2004.04338.x

Li, H. Q., Matsuda, I., Fujise, Y., \& Ichiyama, A. (1999). Short-chain acyl-coA-dependent production of oxalate from oxaloacetate by Burkholderia glumae, a plant pathogen which causes grain rot and seedling rot of rice via the oxalate production. Journal of Biochemistry, 126, 243-253. http://dx.doi.org/10.1093/oxfordjournals.jbchem.a022429.

Lim, J. Y., Lee, T. H., Nahm, B. H., Choi, Y. D., Kim, M., \& Hwang, I. (2009). Complete genome sequence of Burkholderia glumae BGR1. Journal of Bacteriology, 11, 3758-3759. http://dx.doi.org/10.1128/JB.00349-09

Maeda, Y., Shinohara, H., Kiba, A., Ohnishi, K., Furuya, N., Kawamura, Y., ... Hikichi, Y. (2006). Phylogenetic study and multiplex PCR-based detection of Burkholderia plantarii, Burkholderia glumae and Burkholderia gladioli using gyrB and rpoD sequences. International Journal of Systematic and Evolutionary Microbiology, 56, 1031-1038. http://dx.doi.org/10.1099/ijs.0.64184-0

Majerczyk, C., Kinman, L., Han, T., Bunt, R., \& Greenberg, E. P. (2013). Virulence of Burkholderia mallei quorum sensing mutants. Infection and Immunity, 81, 1471-1478. http://dx.doi.org/10.1128/IAI.00048-13

McClean, K. H., Winson, M. K., Fish, L., Taylor, A., Chhabra, S. R., Camara, M., ... Williams, P. (1997). Quorum sensing and Chromobacterium violaceum: exploitation of violacein production and inhibition for the detection of $N$-acylhomoserine lactones. Microbiology, 143, 3703-3711. http://dx.doi.org/10.1099/00221287-143-12-3703

Morohoshi, T., Inaba, T., Kato, N., Kanai, K., \& Ikeda, T. (2004). Identification of quorum-sensing signal molecules and the LuxRI homologs in fish pathogen Edwardsiella tarda. Journal of Bioscience and Bioengineering, 98, 274-281. http://dx.doi.org/10.1016/S1389-1723(04)00281-6

Morohoshi, T., Kato, M., Fukamachi, K., Kato, N., \& Ikeda, T. (2008). N-Acylhomoserine lactone regulates violacein production in Chromobacterium violaceum type strain ATCC 12472. FEMS Microbiology Letters, 279, 124-130. http://dx.doi.org/10.1111/j.1574-6968.2007.01016.x

Morohoshi, T., Nakamura, Y., Yamazaki, G., Ishida, A., Kato, N., \& Ikeda, T. (2007). The plant pathogen Pantoea ananatis produces $N$-acylhomoserine lactone and causes centre rot disease of onion by quorum sensing. Journal of Bacteriology, 189, 8333-8338. http://dx.doi.org/10.1128/JB.01054-07 
Nandakumar, R., Shahjahan, A. K. M., Yuan, X. L., Dickstein, E. R., Groth, D. E., Clark, C. A., ... Rush, M. C. (2009). Burkholderia glumae and B. gladioli cause bacterial panicle blight in rice in the southern United States. Plant Disease, 93, 896-905. http://dx.doi.org/10.1094/PDIS-93-9-0896

Nierman, W. C., Deshazer, D., Kim, H. S., Tettelin, H., Nelson, K. E., Feldblyum, T., ... Fraser, C. M. (2004). Structural flexibility in the Burkholderia mallei. Proceedings of the National Academy of Sciences of the United States of America, 101, 14246-14251. http://dx.doi.org/10.1073/pnas.0403306101

Reece, K. S., \& Phillips, G. J. (1995). New plasmids carrying antibioticresistance cassettes. Gene, 165, 141-142. http://dx.doi.org/10.1016/0378-1119(95)00529-F

Romero, C. M., Deshazer, D., Feldblyum, T., Ravel, J., Woods, D., Kim, H. S., ... Nierman, W. C. (2006). Genome sequence alterations detected upon passage of Burkholderia mallei ATCC 23344 in culture and in mammalian hosts. BMC Genomics, 7, 228. http://dx.doi.org/10.1186/1471-2164-7-228

Sambrook, J., Fritsch, E. F., \& Maniatis, T. (1989). Molecular cloning: a laboratory manual (2nd ed.). Cold Spring Harbor, NY: Cold Spring Harbor Laboratory Press.

Shaw, P. D., Ping, G., Daly, S. L., Cha, C., Cronan, J. J., Rinehart, K. L., \& Farrand, S. K. (1997). Detecting and characterizing $N$-acylhomoserine lactone signal molecules by thin-layer chromatography. Proceedings of the National Academy of Sciences of the United States of America, 95, 6036-6041. http://dx.doi.org/10.1073/pnas. 94.12.6036

Simon, R., Quandt, J., \& Klipp, W. (1983). A broad host range mobilization system for in vivo genetic engineering: transposon mutagenesis in Gram-negative bacteria. Nature Biotechnology, 1, 784-791. http://dx.doi.org/10. $1038 /$ nbt1183-784

Tsushima, S. (1996). Epidemiology of bacterial grain rot of rice caused by Pseudomonas glumae. Japan Agricultural Research Quarterly, 30, 85-89.

Tsushima, S., Naito, H., \& Mogi, S. (1991). Reduction of virulence and colonial variation of Pseudomonas glumae cultured on PSA medium. Bulletin of the Kyushu National Agricultural Experiment Station, 26, 361-379 (in Japanese with English summary).

Tsushima, S., Tsuno, K., Mogi, S., Wakimoto, S., \& Saito, H. (1987). The multiplication of Pseudomonas glumae on rice grains. Annals of the Phytopathological Society of Japan, 53, 663-667 (in Japanese with English summary).

Uematsu, T., Yoshimura, D., Nishiyama, K., Ibaraki, T., \& Fujii, H. (1976). Occurrence of bacterial seedling rot in nursery flat, caused by grain rot bacterium Pseudomona glumae. Annals of the Phytopathological Society of Japan, 42, 310-312.

Ulrich, R. L., DeShazer, D., Hines, H. B., \& Jeddeloh, J. A. (2004). Quorum sensing: a transcriptional regulatory system involved in the pathogenicity of Burkholderia mallei. Infection and Immunity, 72, 6589-6596. http://dx.doi.org/10.1128/IAI.72.11.6589-6596.2004

Yoshida, S., Hiradate, T., Tsukamoto, K., Hatakeda, K., \& Shirata, A. (2001). Antimicrobial activity of culture filtrate of Bacillus amyloliquefaciens RC-2 isolated from mulberry leaves. Phytopathology, 91, 181-187. http://dx.doi.org/10.1094/PHYTO.2001.91.2.181

Zeigler, R. S., \& Alvares, E. (1989). Grain discoloration of rice caused by Pseudomonas glumae in Latin America. Plant Disease, 73, 368. http://dx.doi.org/10.1094/PD-73-0368B

\section{Copyrights}

Copyright for this article is retained by the author(s), with first publication rights granted to the journal.

This is an open-access article distributed under the terms and conditions of the Creative Commons Attribution license (http://creativecommons.org/licenses/by/3.0/). 